\title{
What is the Most Precise Endoscopic Finding for Predicting the Clinicopathological Behaviors in Ulcerative Early Gastric Cancer?
}

\author{
Youngdae Kim \\ Division of Gastroenterology and Hepatology, Department of Internal Medicine, Chosun University Hospital, Gwangju, Korea
}

See "Endoscopic Factors that Can Predict Histological Ulcerations in Early Gastric Cancers" by Jaesin Lee, Byung-Wook Kim, Cheal Wung Huh, et al., on page 328-333.

According to its definition, early gastric cancer (EGC) invades only into the mucosa or submucosa, regardless of lymph node (LN) involvement or distant metastasis. Endoscopic resection can provide curative treatment in cases with a negligible probability of $\mathrm{LN}$ metastasis. The absolute indication is differentiated-type adenocarcinoma less than $2 \mathrm{~cm}$ in diameter without the presence of an ulcer or ulcer scar. En bloc resection becomes possible irrespective of lesion characteristics in the era of endoscopic submucosal dissection (ESD). The indications have been expanded based on surgical specimens reviews by Gotoda et al. ${ }^{1}$ Nowadays, ESD has been established as an important treatment option for selected cases of EGC. Therefore, accurate preprocedural endoscopic evaluation is essential for establishing an appropriate treatment strategy for EGC. The ulcerative mucosal cancer smaller than $3 \mathrm{~cm}$ of the differentiated type, which was one of previous expanded indications of ESD, are now defined as the absolute indication of ESD. ${ }^{2}$ This indication is also defined as endoscopic curability A (eCuraA) after ESD. ${ }^{2}$

An ulcerative finding in EGC is one of the important factors for the feasibility of ESD due to the limitation for prepro-

\footnotetext{
Received: April 22, 2020 Accepted: May 18, 2020

Correspondence: Youngdae Kim

Division of Gastroenterology and Hepatology, Department of Internal Medicine, Chosun University Hospital, 365 Pilmun-daero, Dong-gu, Gwangju 61453, Korea Tel: +82-62-220-3364, Fax: +82-62-224-5494, E-mail: giydkim@chosun.ac.kr ORCID: https://orcid.org/0000-0003-0896-2135

(c) This is an Open Access article distributed under the terms of the Creative Commons Attribution Non-Commercial License (http://creativecommons.org/ licenses/by-nc/3.0) which permits unrestricted non-commercial use, distribution, and reproduction in any medium, provided the original work is properly cited.
}

cedural endoscopic evaluation. The decisive issues are the ambiguous definition of ulcerative findings on endoscopy and discrepancies between endoscopic findings and histologic diagnoses. A histological ulcer is defined as a defect that extends deep into the submucosal or deeper layers of the gastrointestinal wall. Typical ulcerative findings are converging folds, deformity of the muscularis propria, and fibrosis in the submucosa or deeper layers on the Gotoda's review. ${ }^{1}$ The histological finding of an ulcer is considered an important factor in the treatment decision for EGCs and the curability of ESD. It should also be distinguished from a biopsy-derived scar. $^{2}$ Although a mucosal defect alone cannot be defined as an ulceration, it may be described as an endoscopic ulcer by not only beginners but also experienced endoscopists.

The careful examination and accurate evaluation of endoscopic ulcerations is necessary to avoid unnecessary surgery due to the overestimation of ulcerative EGCs. Park et al. reported the discrepancy in endoscopic ulcerations among endoscopists according to the difference in the experience of endoscopic exam and ESD. ${ }^{3}$ In a survey with 7 endoscopic images and questionnaires via e-mail, the diagnostic accuracy was $28.2 \%$ in the case of a shallow mucosal break without converging folds. Although this case was not a pathologic ulceration, $71.8 \%$ of all respondents answered that it was an ulcerative EGC. The diagnostic accuracy was also low in the pathologically ulcerative case of a depressed lesion with converging folds without a mucosal break, only $35.9 \%$ of all respondents diagnosed this as ulcerative EGCs. Lee et al. suggested that converging folds with color change on endoscopic examination were statistically significant factors for predicting 
histological ulceration. ${ }^{4}$ Converging folds and color changes have been shown to be independent factors predicting histological ulcerations.

Much like the overestimation of mucosal breaks, endoscopists must also beware of the underestimation of EGCs. Endoscopic ulcers present as various shapes and morphologies in the malignant cycle. ${ }^{5}$ This may lead to the delayed diagnosis of malignancy or the misdiagnosis in $\mathrm{T}$ staging. The precise preprocedural $\mathrm{T}$ staging is essential to decide the treatment modality of ulcerative EGCs. ESD has been accepted as a treatment of ulcerative intramucosal EGCs with a very low risk of LN metastasis. However, if the malignant lesion invades into the submucosal layer, ESD should not be performed due to the high risk of LN metastasis. Kim et al. reported that the rate of LN metastasis in ulcerative EGC was overall 15.1\%. ${ }^{6}$ There were no cases of LN metastasis in the mucosa-confined lesions, but the rate of LN metastasis in submucosa-infiltrating ulcerative EGCs was 28.2\%. ${ }^{6}$ Lymphovascular invasion was the only significant risk factor of LN metastasis on multivariate analysis. The diagnostic accuracy of conventional endoscopy and the diagnostic yield of endoscopic ultrasonography on ulcerative EGC are limited compared with those on non-ulcerative EGCs. ${ }^{7,8} \mathrm{LN}$ metastasis was reported in $15.8 \%$ of SM1 invasive differentiated cancer. ${ }^{9}$ Submucosal invasion was independently associated with endoscopic findings such as type III ulcers, tumorous bank, a fusion of converging folds, hardness or decreased flexibility, and incomplete ulcer healing on follow-up endoscopy. In the study of the association of ulcer stage with clinical behaviors in EGCs, ${ }^{10}$ it was shown that the endoscopic stage of ulcers may predict the clinicopathological behaviors of EGC. The active ulcer stage was one of the independent risk factors for LN metastasis.

In conclusion, ESD is an acceptable therapeutic modality for ulcerative EGCs. For curative endoscopic resection, precise endoscopic evaluation of the lesion is essential. The biggest issue is that many endoscopists may overestimate endoscopic mucosal breaks as histologic ulcers, which may result in more aggressive and unnecessary surgery. The converging fold is one of the most precise endoscopic factors for the prediction of histological ulcers. Mucosal discoloration is also another important factor for the prediction of histological ulcers. The surrounding mucosal fold changes including tumorous bank formation, fusion of converging folds, and marked hardness or decreased flexibility of surrounding folds suggest submucosal cancer invasion. ESD should not be performed in these lesions due to the high risk of LN metastases. Therefore, endoscopists should make an accurate diagnosis of histological ulcer and prediction of accurate $\mathrm{T}$ staging through the endoscopic findings, even if this is difficult for experienced endoscopists.

Conflicts of Interest

The author has no financial conflicts of interest.

\section{REFERENCES}

1. Gotoda T, Yanagisawa A, Sasako M, et al. Incidence of lymph node metastasis from early gastric cancer: estimation with a large number of cases at two large centers. Gastric Cancer 2000;3:219-225.

2. Japanese Gastric Cancer Association. Japanese gastric cancer treatment guidelines 2018 (5th edition). Gastric Cancer 2020 Feb 14 [Epub]. https://doi.org/10.1007/s10120-020-01042-y.

3. Park SM, Kim BW, Kim JS, Kim YW, Kim GJ, Ryu SJ. Can endoscopic ulcerations in early gastric cancer be clearly defined before endoscopic resection? A survey among endoscopists. Clin Endosc 2017;50:473-478.

4. Lee J, Kim BW, Huh CW, Kim JS, Maeng LS. Endoscopic factors that can predict histological ulcerations in early gastric cancers. Clin Endosc 2020;53:328-333

5. Sakita T, Oguro Y, Takasu S, Fukutomi H, Miwa T. Observations on the healing of ulcerations in early gastric cancer. The life cycle of the malignant ulcer. Gastroenterology 1971;60:835-839 passim.

6. Kim JW, Lee H, Min YW, et al. Oncologic safety of endoscopic resection based on lymph node metastasis in ulcerative early gastric cancer. J Laparoendosc Adv Surg Tech A 2019;29:1105-1110.

7. Akashi K, Yanai H, Nishikawa J, et al. Ulcerous change decreases the accuracy of endoscopic ultrasonography diagnosis for the invasive depth of early gastric cancer. Int J Gastrointest Cancer 2006;37:133-138.

8. Kim GH, Park DY, Kida M, et al. Accuracy of high-frequency catheter-based endoscopic ultrasonography according to the indications for endoscopic treatment of early gastric cancer. J Gastroenterol Hepatol 2010;25:506-511.

9. Lee JI, Kim JH, Kim JH, et al. Indication for endoscopic treatment of ulcerative early gastric cancer according to depth of ulcer and morphological change. J Gastroenterol Hepatol 2012;27:1718-1725.

10. Lee YJ, Kim JH, Park JJ, et al. The implications of endoscopic ulcer in early gastric cancer: can we predict clinical behaviors from endoscopy? PLoS One 2016;11:e0164339. 